\title{
Insuficiencia renal crónica en la infancia. Encuesta nacional
}

\author{
Anita Valenzuela L.' ; Edda Lagomarsino F. ${ }^{2}$; Felipe Cavagnaro SM. ${ }^{2}$; Eric Solar G.'
}

\section{Chronic renal failure in chilean cbildren: a national survey}

\begin{abstract}
A national survey on chronic renol falure CCRFy in chiidren was carried ouk by year 1994 by the branci of Neshrology of the Chilean Pediaric Sociery, and 194 potients with CR- were recorded lo p:evolence of 39.6 per million inhotitorils 18 vears of age or less). $70.3 \%$ lived of Santiago, the capita ciry of the country, $54 \%$ were female, $63.4 \%$ were older than :0 years ard $2.3 \%$ were under 2 years of age. The most frequent causes of renal failure were ur nary mact cbsiructions $178.6 \%$, glomeruiar oiseases [18\% and renal fisease seconday to urinary reflux $: 17.5 \%$. At the lime of report, $57.2 \%$ of cases were under medical (nos diaiytic! monacement, $16.5 \%$ iwere in a dialysis piogram and orly $20.3 \%$ had been given a rerial ranspiant. Perifa"eol procedures were usec in only 7 (3,6\%) of 32 palienis in dialysis, in controst to othe- countries where they ore by $r a$ the prefered meshods for childen less than $20 \mathrm{~kg}$ weignt. Among renal ransplant patients: $57 \%$ were reportec lo be in good renal furiction cnd crily $10 \%$ were back on diclysis. Of 21 chidren less then five years old, only one was on dalysis and no cne had peen gueri a rena Irarsplanit.
\end{abstract}

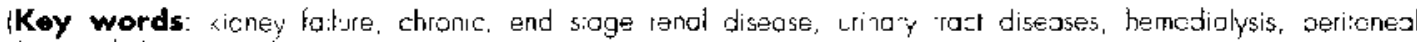
divysis, kidney lonsplantaton.;

La insuficjencia renal crónica (IRC) es la limitación y reducción progresiva de la función homeostática del riñón, por un período mayor a tres meses, alterando el equilibrio del medio interno en magnitudes que pueden llegar a ser incompatibles con la vida. El continuo progreso de los tratamientos destinados a reemplazar las funciones renales ha producido notoria incjoria en cl pronóstico de estos enfermos. La sobrevivencia de miles de pacientes en IRC terminal ha sido prolongada y la calidad de vida mejorada con la aplicación de los programas de diálisis y trasplante, a partir de 1959 I.

Los estudios epidemiologicos han sido esenciales para planificar programas de reemplazo de la función renal. En Chile, la Sociedad Chilena de Nefrología ha llevado. desde 1980, un registro anual de adultos en hemodiálisis crónica, mientras la Rama de Nefrología de la Sociedad Chilena de Pcdiatría realizó una primera en-

1. Servicio de Pediatría. Unidad de Nefrología, Hospital Sótéro del Río.

2. Departamento de Pediatría, Unidad de Nefrología, Escue. ha de Medicina Pontificia Universidad Católica de Chile cuesta sobre neftópatas crónicos en 1989 , que permitió identificar 112 pacientes solo en los hospitales de la Región Metropolitana, que corresponde a la ciudad capital del país y sus alrededores ${ }^{2}$. La necesidad de aplicar criterio estándar y actualizar la información, aumentando la cobertura, nos impulsó a realizar una encuesta nacional sobre niños con insufticiencia renal cróniça en el año 1994.

\section{Material y Método}

En mayo de 1994 se envió a todos los miembros de la Rama de Nefrología de la Sociedad Chilera de Pediatría y a los servicios de pediatría del pais, una encuesta codificada requiriendo información sobre los pacientes vivos menores de 18 afios con IRC, a esa fecha.

Se defínió como insuticiencia renal crónica la de todo paciente cuya concentración de creatinina plasmática fueș Jos o más veces milyor que la normal para edad y sexo o cuya filtración glomerular fuera inferior a $30 \mathrm{ml} / \mathrm{min}$. $1,73 \mathrm{~m}^{2}$. Se consideró insuficiencia renal crónica terminal la concentración de creatinina plasmática cuatro o más vetes superior a ta normal para edad y sexo o la filtración gloinerular menor de $15 \mathrm{ml} / \mathrm{minn} \cdot 1,73 \mathrm{~m}^{2} 3$ al menos dutante tres meses, de esta manera se evitó incluir pacientos con insuficiencia renal aguda. 
La encuesta incluía información sobre identiticación. fecha de nacimiento, sexo. dingnóstico etiologico, otros diugnósticos rełacionados. función retal quitrógenn ureico en sangre, creatinina plasmatica o aclacamiculo de creatjnjon) al monento del estudio. biopsia remal con su diagnóstico histológico, tratamiento recibıdo y estado actual del paciente. Para clasiffear ha enfennedad renal primaria se utilizó la clasiticación de Esbjömert. En el grupo de hipoplasia-displasia y obstrucción se incluyeron niños con agenesia. nefreciornía precoz o riñón displásico unilateral con obstrucción de la uretra o del utéter contralateral. E) sindrome de abdomen en pruna ("prume belly") y la vejiga neurogénica fueron incluidos entre las enfermedades obstructivas debido al obstículo funcional que ocurre en ellos. Los pacientes con reflujo vesicoureteral se registraron corno pielonefritis cronica o nefropatia por reflujo y no, como en la clasificación de Habibs", entre las malformaciones. En la definición de nefropatía del reflujo se exjgió que hubiese imágenes radiológicas de cicatrices renales asociadas a antecedente de infección del Iracto trinario (ITU) con o sin reflujo vesicoureteral en el momento del diagnóslico.

Según el tratamiento, los pacientes se clasificaron entre los que habian recibido trasplante renal. independientertente de su manejo acrual: los que estaban en dialisis y no habian sido sometidos a transplante de riñón y los qut recibían sólo 1ratamienı médico.

La prevalencia se calculó dividiendo el núnero de ninos menores de 18 años que cumplían el criterio de IRC. por el número de niños dé esa misma categoría de edad. según el cesso nacional de población del año $1992^{\prime}$.

\section{Resultados}

La encuesta fue respondida por trece centros pediátricos, siete de la Región Metropolitana. Se contabilizaron 243 pacientes, que se redujeron a 194 casos, después de descartar 8 casos de doble registro (ubicando a cada paciente en el centro donde se hizo inicialınente el diagnóstico de IRC), 11 niños fallecidos, 20 enfermos por no cumplir con los criterios de IRC, 7 pacientes por información insuficiente para el análisis y 3 por tener más de 18 años de edad. La prevalencia de IRC fue de 39,6 por millón de habitantes menores de 18 años; $77,4 \%$ habían sido diagnosticados en 1a Región Metropolitana; $103(53 \%)$ eran mujeres; $123(63,4 \%)$ tenían 10 años de edad o más, 27 (14\%) eran menores de 5 años y $5(2,3 \%)$ menores de 2 años. El número de casos aumentaba con la edad, salvo en el grupo etario mayor de 15 años.

Las causas más frecuentes de IRC fueron: uropatía obstructiva $(18,6 \%)$; glomerulopatías (18\%), nefropatia por reflujo $(17,5 \%)$ y displasia renal $(15,5 \%)$, siendo las individuales más importantes nefropatía de reflujo $(17,5 \%)$; veji- ga neurogénica $(11,3 \%)$; glomeruloesclerosis focal y segmentaria $(6,7 \%)$. enfermedad poliquística $(6,7 \%)$ y síndrome hemolítico urémico (5.2\%). En 8,8\% de los casos no se identiticó la etiología de la IRC (tabla 1).

El tratamiento recibido (tabla 2) fue médico en $57,2 \%$ (11) de 194), 16,5\% (32 de 194) estaba en diálisis sin haber recibido trasplante y a $26,3 \%$ (51 de 194) se les había practicado trasplante en al menos una oportunidad. Trece

Tabla 1

Etiología de la insuficiencia renal crónica en niños de trece centros chilenos

\begin{tabular}{|c|c|c|c|}
\hline \multirow[b]{2}{*}{ Etiología } & \multicolumn{3}{|c|}{ Ni iñs } \\
\hline & $\mathbf{n}$ & $\mathbf{n}$ & $\%$ \\
\hline Hipodisplasia & 30 & & 15,5 \\
\hline Hipodisplasia y obstrucción & 7 & & 3,6 \\
\hline $\begin{array}{l}\text { Obstruclivas } \\
\text { Valvas uretrales } \\
\text { Estenosis ureterales bilaterales } \\
\text { Otras malformaciones obstructivas } \\
\text { Síndrome de abdomen en prena } \\
\text { Vejiga neurogénica }\end{array}$ & 36 & $\begin{array}{r}6 \\
1 \\
2 \\
5 \\
22\end{array}$ & 18,6 \\
\hline $\begin{array}{l}\text { Glomerulopatias } \\
\text { G. esclerosis focal y segmentaria } \\
\text { GN con esclerosis difusa avanzada } \\
\text { GN Inesangio capilar } \\
\text { GN rápidamente progresiva } \\
\text { Nefropalía por IgA } \\
\text { Nefropalía lúpica } \\
\text { Síndrome de Schönlein Henoch } \\
\text { GN no clasificada }\end{array}$ & 35 & $\begin{array}{r}13 \\
2 \\
4 \\
1 \\
2 \\
2 \\
3 \\
8\end{array}$ & 18,0 \\
\hline $\begin{array}{l}\text { Defectos hereditarios del riñón } \\
\text { Nefromoptisis juvenil } \\
\text { Enfermedad poliquística } \\
\text { Síndrome nefrótico congênito } \\
\text { Sindrome de Alport }\end{array}$ & 18 & $\begin{array}{r}3 \\
13 \\
1 \\
1\end{array}$ & 9,3 \\
\hline $\begin{array}{l}\text { Nefropatías vasculares } \\
\text { Sindrome hemolítico urémico } \\
\text { Asfixia neonalal } \\
\text { Takayasu }\end{array}$ & 12 & $\begin{array}{r}10 \\
1 \\
1\end{array}$ & 6.2 \\
\hline $\begin{array}{l}\text { Nefropatía del refiujo } \\
\text { Pielonefritis }\end{array}$ & 34 & & 17,5 \\
\hline $\begin{array}{l}\text { Otras enfermedades renates } \\
\text { Síndrone de Drash } \\
\text { Vefrilis intersticial } \\
\text { Síndrome de Fanconi } \\
\text { Diabetes insípida }\end{array}$ & 5 & $\begin{array}{l}1 \\
2 \\
1 \\
1\end{array}$ & 2.6 \\
\hline No clasificadas & 17 & & 8.8 \\
\hline
\end{tabular}

G: glomérulo; N: nefritis 
Tabla 2

Trataniento empleado en 194 niños con insuliciencia renal crónica en trece centros de salud chilenos

\begin{tabular}{|c|c|c|c|}
\hline Etiología & II & $\underset{n}{N \mathbf{i} n}$ & * \\
\hline Médico & 111 & & 57.2 \\
\hline Crealinısa 2N & & 51 & \\
\hline Crcatinina 4N & & 47 & \\
\hline Otros valores & & 13 & \\
\hline Diálisis & 32 & & 16.5 \\
\hline Hemodiâlisis & & 25 & \\
\hline Péritoneadiálisis & & 7 & \\
\hline Trasplante & 51 & & 26,3 \\
\hline Función renal norstat & & 28 & \\
\hline Funtion renol alrerada & & 17 & \\
\hline En diálisis & & 5 & \\
\hline Sin control & & 1 & \\
\hline Tatal & 194 & & 100.0 \\
\hline
\end{tabular}

N: valor nomal de creacimina para el sexo y la edad

niños en tratamiento médico no sc habían controlado en los últimos seis meses. desconociéndose su creatininemia en el momento del estudio. De los 32 pacientes en diálisis $(16,5 \%)$, en 7 el procedimiento cra peritoneal (3.6\%). Súlo uno de los 21 pacientes menores de 5 años estaba siendo dializado y ninguno había sido beneficiado de trasplante, a pesar que en 10 había indicación absoluta de sustitución renal porque la creatinina sérica era 4 o más veces lo normal para edad y sexo. Los 5 pacientes menores de 2 años estaban en tratamiento médico, aunque al menos dos necesitaban tratamiento de sustitución.

De los 51 pacientes en que se había hecho trasplantes, $28(57 \%)$ tenjan buena función rênal, $5(10 \%)$ cstaban nucvamente con diálisis y en $17(33 \%)$ ia función renal eslaba alterada en el momento del estudio y requerían un nucvo trasplante o entrar en un programa de diálisis.

\section{Comentario}

El propósito de actualizar y mejorar la información sobre los pacientes pediátricos con IRC? se logró al aumentar el número de casos registrados de 112 a 194. Este incremento puede ser debido a mayor interés en responder la cncueslat, especialmente de los hospitales de las regiones, como tambićn a aumcrito real del número de pacientes, dada la mayor sobrevida de estos niños en la actualidad. Sin embargo, el registro insuficiente es un problema siempre asociado a este tipo de estudios.

No fue posible calcular la incidencia de insuficiencia renal crónica en esta serie porque no se consignaton los casos nuevos anuales, que se estiman, en el mundo, en 4 o 5 por millón de habitantes menores de 18 años. De estos nuevos casos anuales, aproximadamente la mitad llega a sufrir insuficiencia renal crónica terminal? ${ }^{7}$. La prevalencia de 39.6 por millón de habitantes menor de 18 años en nuestro estudio, es comparable con la de 37,0 del Registro Europeo de Enfermedades Renales (ESRD) en $1990^{8}$ y la de Lorreine (años 1975 a 1990) que mostró fluctuaciones entre 15.8 y 64.7 , con 37.0 por millon para $1990^{\circ}$. La tasa de prevalencia debería seguir aumentando en el futuro próximo, aunque la incidencia se manluviese constante, debido a la disminución en la tasa de mortalidad de los pacientes con el tratamiento ${ }^{1 t}$. La solución al problema debe enfocarse probablemente en medidas preventivas primarias, tendientes a evital las causas de falla renal, y secundarias, tendientes a limitar la progresión del daño después que la injuria renal se ha producido. La concentración de los principales recursos para el manejo de reemplazo de la función renal infantil en la Región Metropolisana de Santiago, explica que. a pesar de vivir en ella alrededor de un tercio de los habitantes del país, se registra en clla $77,4 \%$ de los casos detectados de IRC en niños.

Las causas etiológicas de IRC no han variado, en Chile, durante el tiempo comprendido entre las dos encuestas. Hay una disminución en el porcentaje de las pielonefritis con o sin reflujo vesicoureleral desde $44 \%$ a $18 \%$, pero como la forma de clasificarlas cambió, no se pueden salcar conclusiones definitivas de esta diferencia. Entre las causas prevenibles de IRC, la pielonefritis representa $6 \%$ de los casos en Suecia ${ }^{4}$. la cifra más baja en Europa, y en Chile $18 \%$, Io que difícilmente puede ser explicado por diferencias metodologicas, sino más bien por la prococidad con que se detectan en el primer país los problemas renales, gracias a pro- 


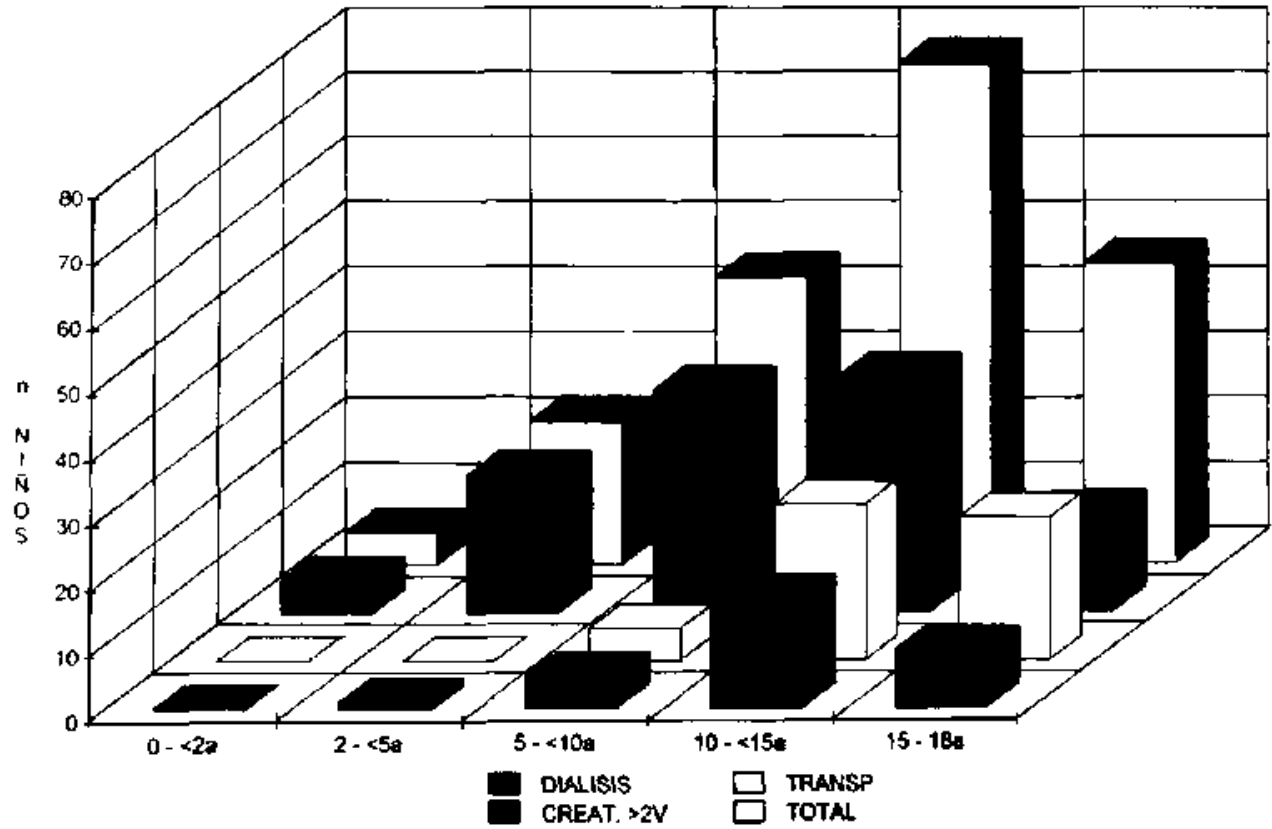

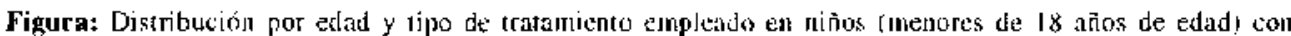
insuficiencia renal crónicia.

gramas de salud que cubren las clínicas de niños sanos y los colegios, a la cuidadosa investigación y al seguimiento de que son objeto los niños con infecciones urinarias. La proporción debiera incjorar en las próximas cncuestas nacionales, pues ha aumentado la disponibilidad $y$ disminuido el coste de los estudios de imágenes necesarios para su manejo. Tambicn la vejiga neurogénica es menos frecuente en el primer caso $(1,4 \%)$ que en el segundo $(1,3 \%)$ sugiricndo la necesidad de apoyar toda medida tendiente a evitar y tratar estas causas susceptibles de prevencion.

El tratamiento ideal de la insuficiencia rcnal crónica es el trasplante, en cspecial para los niños. Sin embargo, sólo un cuarto de los afectados incluidos habian recibido este beneficio al momento de la encuesta, iodos mayores de 5 años. Esta baja proporción se explica, en parte, por fallas en la infraestructura, apoyo económico y, especialmente. dificultades de base sociocultural para la doneción de órganos.

Para los niños que esperan un trasplante, que no pueden recitirlo por existir contraindicaciones o aquellos en que éste ha fracasado, la única alternativa de sobrevida se basa en la diálisis. La hemodiálisis, método de depuración sangui- nea más usado en adultos, es poco recomendable en pacientes cuyo peso corporal sea inferior a $20 \mathrm{~kg}$, debido a que produce bruscas variaciones del volumen y composición sanguíneos y el acceso vascular es técnicamente difícil. La peritoneodiálisis es c] método preferible en los niños y el único posible para recién nacidos y lactuntes menores con nefropatía crónica; puede hacerse en el hogar del paciente. no requiere supervision directa, reduce el riesgo de los trastornos mencionado para la hemodiálisis y desde hace décadas se emplea ampliamente en otros países, como México, donde más de $90 \%$ de los pacientes con insuficiencia renal crónica terminal están en programas de diálisis peritoneal crónica.

Hasta el año 1994, la peritoneodialisis cronica se realizaba en Chile con dificultades de infraestructura y equipamiento que inhibían su empleo porque habia sido omitida de las prestaciones del Fondo (público) Nacional de Salud y de las Instituciones (privadas) de Salud Previsional, que sin cmbargo cubrían los gastos de hemodiálisis. La Rama de Nefralogía de la Sociedad Chilena de Pediatría, consciente de cste problema, solicitó y obtuvo Jel Ministerio de Salud la inclusión de la peritoneodiálisis entre 
esas prestaciones. Los resultados de esta encuesta constituyeron parte importante de los antecedentes entregados al Ministerio, al delinear el número de pacientes y demostrar la indefensión de los niños menores de 5 años con IRC en el país. Desde el 1 de enero de 1995 la peritoneodiálisis continua en pacientes crónicos menores de 13 años se incorporó al listado de prestaciones del Fondo Nacional de Salud con el código TEN 19-0I-026. En las próximas encuestas cabría esperar un aumento del número de nir̃os en diálisis peritoneal. demostrando la utilidad de la medida en la IRC. especialmente en los menores de cinco años, en mejorar la sobrevida y facilitar la preparación de estos niños para el trasplante renal.

Como estrategia sectorial de salud pública, parece necesario apoyar el desarrollo de centros que ofrezcan peritoneodiálisis, como asimismo a los programas de educación destinados a mejorar la oferta de órganos para trasplantes.

\section{Resumen}

En una encuesta nacional tealizada en 1994 sobre insuficiencia renal crónica en niños se registraron 194 casos (prevalencia de 39,6 por miJlón de habitantes menores de 18 años); $54 \%$ mujeres; $77,3 \%$ de la Región Metropolitana; $63,4 \%$ mayores de 10 años y $2,3 \%$ menores de 2 años. Las causas más frecuentes de la insuficiencia renal eran las uropatías obstructivas $(18,6 \%)$, las enfermedades glomerulares (18\%) y las nefropatias por reflujo $(17,5 \%)$. El tratamiento, al momento de la encuesta, era médico en $57,2 \%$ de los casos, con diálisis en $16,5 \%$ y sólo $26.3 \%$ había sido beneficiado con un trasplante renal. En sólo $7(3,6 \%)$ de 32 niños en diâlisis $(3.6 \%)$ ésta era peritoneal en contraste con 10 que sucede en otros países, donde se la prefiere en los pacientes cuyo peso corporal es menor que $20 \mathrm{~kg}$. De los niños que recibieron trasplante. $57 \%$ tenían buena función renal y sólo $10 \%$ habían vuclto a necesitar diálisis. De 2l menores de cinco años sólo uno está en diá- lisis y ninguno ha recibido un irasplante. Con esta información se respaldó y obluvo la inclusión de la diálisis peritoneal entre las prestaciones del Fondo (público) Nacional de Salud.

(Palabras clave: insuficiencia renal crónica, enfermedad renal terminal, enfermedades de la vía urinaria, hemodiálisis, peritoneodiálisis, trasplante renal.)

\section{Agradecimientos}

A los médicos de los huspilales de Calama, La Serena, Clíntca Las Condes. Clínico de la Pontificia Universidad Católıa, Excquiel Gonzälez Cortés. Luis Calvo Mackenna, Roberto del Río, San Juan de Dios. Sótero del Rio, Chillán. Concepcion, Valdivia y Coihaique, su colaboración al suministrar la información que permitió realizar este trabajo.

\section{Referencias}

1. Potter D. Holliday MA, Piel CF, ef al: Treatment of end stage remal disease in children: A 15 years experience. Kidney [ 1980; 18: 103-109

2. Delacefi A, Cono $F$ : Insuficiencia renal crónica. Rev Clis Pediatr 1990; 61 (Supl. 1): 19-20.

3. Schwartz GF. Haycoke GB, Spitzer A: Plasma creatinine and urea concentration in children: Normal $v$ alues for age and scx. J Pediats 1976; 88: 828-830.

4 Esbjimer $E$. Aronson S. Berg U. Jodal U. Linne T: Chitdren with chronic renal fajlure in Sweden 19781985. Pediatr Nephrol 1990; 4: 249-252.

5. Habib R. Brover M. Betunarz M: Chonic renal failure in children. Causes, rate of deterioration and survival data. Nephron 1973: 11:209-220.

6. Lusritumo Nacionetl de Estadisticas: Censo de Pobla. ción y Virienda, Chile J992. Imprenta Alborada S.A. Condinadora General Edicion M. Zajer A. 1992: 69

7. Zilleraeto $G$ : Diálisis peritoneal crónica en el niño. Rev Chil Pediatr 1982;53:603-606.

8. Bumier $F$. Brover $M$, Bryger $M$. ef al: Denography of dialysis and transplantation in children in Europe 1985. Report from the Eutopean Dialysis and Transplant Associarion Registry. Neptrol Dial Transplant 1988; 3: 235-243.

9. Deteat J. Andre J. Briancon S, Musse J. Chronic renal fuilure in children: an epideniological survey in Lorraine (France) 1975-1990. Pediatr Nephrol 1994; 8: $472-476$

10. Port F: End stage renal disease: magnitude of the problem, progtrosis of future trends and possible solutions. Kidney 1 199.5: 48 is !: 3-6. 